\title{
Dot Line Pattern Formation in Photoresist Films by Mask-Guided LIPSS Formation Due to Excimer Laser Irradiation
}

\author{
Shengying Lai ${ }^{* * *}$, Pierre Lorenz ${ }^{* *}$, Martin Ehrhardt ${ }^{* * * * *}$, Bing $\operatorname{Han}^{* * *}$, Jian Lu, Klaus Zimmer ${ }^{* *}$ \\ * School of Science, Nanjing University of Science \& Technology, Xiaolingwei 200, Nanjing 210094, \\ China \\ ** Leibniz Institute of Surface Engineering (IOM), Permoserstr. 15, 04318 Leipzig, Germany \\ E-mail: klaus.zimmer@iom-leipzig.de \\ *** Advanced Launching Co-innovation Center, Nanjing University of Science \& Technology, \\ Xiaolingwei 200, Nanjing 210094, China
}

\begin{abstract}
Micro-nano patterns are requested for various applications mimicking structures from nature. Here top-down patterning by phase mask irradiation was combined with bottom-up LIPSS (Laser Induced Periodic Surface Structures) formation processes to achieve hierarchically organized structures. Novolac films (200 to $700 \mathrm{~nm}$ film thickness) were irradiated by a high number of excimer laser pulses $(N=1500)$ at very low fluences $\left(F=12 \pm 1 \mathrm{~mJ} / \mathrm{cm}^{2}\right)$ to achieve the LIPSS formation. The area, the organization and the appearance of the LIPSS are related to the confinement of the LIPSS formation area due to the incident laser energy density distribution at the sample. At particular conditions using phase mask irradiation single chain dot like pattern with a size of approximately $220 \mathrm{~nm}$ have been achieved. This particular result represents a unique feature of LIPSS formation by surface scattering due to the confinement of the laser-driven interaction processes.
\end{abstract}

DOI: $10.2961 /$ jlmn.2019.02.0002

Keywords: laser, excimer laser, novolac, polymer, micro pattern, nano pattern, LIPSS

\section{Introduction}

Submicron pattern fabrication by laser machining is still challenging as the achievable pattern size is related to the optical resolution that is given by the laser wavelength used for direct writing e.g. by laser ablation. Therefore, various approaches of submicron pattern formation by bottom-up processes such as self-assembly or self-organizing processes are studied. Those processes of self-assembly and self-organization enable a fast and easy sub micrometer patterning of surfaces with patterns of a size that are not rigorous determined by the wavelength of the laser irradiation.

Such mechanisms of submicron/nanopatterning formation by laser irradiation, generally called LIPSS (laser-induced surface structures) or ripples (for linear LIPSS) have been observed for laser irradiation of polymers as well. For example, cone-like structure formation has been observed by laser irradiation of polymers with laser fluences near the threshold of ablation. The process of cone structure formation has been explained by masking effects from redeposition products (carbon) or impurities in the material $[1,2]$.

Lazare et al studied the formation of ripples in different polymers due to pulsed laser irradiation and found that by the usage of polarized excimer laser light ripples with a periods 1.3 times smaller than the laser wavelength are formed [3]. Similar mechanism of ripples formation was found by Dyer et al. at VUV (vacuum ultraviolet) irradiation of different polymers. The reported periods were in the range of 100 to $150 \mathrm{~nm}$ and should be related to the interference of the surface scattering of the incoming light at surface irregularities [4].

Linear LIPSS patterns (ripples) were observed during the irradiation of different polymer foils and films with linear polarized nanosecond ultraviolet (ns-UV) laser (248 nm,
$266 \mathrm{~nm}$ ) with high pulse numbers. Usually an extremely low threshold fluence (e.g. $3-5 \mathrm{~mJ} / \mathrm{cm}^{2}$, [5]) for LIPSS formation in polymers was found. Their formation was addressed to the scattering of the incoming laser light at some defects like pit, structural defects or perturbations that forms interference energy density distribution.

The light scattering at rough surfaces that was studied by Sipe [6] results also in components scattered in plane to the surface. The energy density modulation due to the interference of the incidence and the scattered light can influence the particular pattern formation process. During subsequent laser pulses the spatial period of the roughness that provides the strongest feedback mechanism developed to LIPSS finally [6].

Periodic and irregular pattern with periods around $1 \mu \mathrm{m}$ that period changed with the laser fluence were observed by Niino during ablation of amorphous PES (Polyether Sulfones ) with $308 \mathrm{~nm}$ excimer laser [7]. The mechanism was no clear but is not probably related to in plane scattering of the incidence laser light. Self-organizing structures in stretched polymers have been observed for PET ( Polyethylene Terephthalate) after Excimer laser irradiation; the pattern formation is related to the relaxation of the stress in the material $[8,9]$.

It has to be mentioned that surface stress fields can also cause the formation of periodic or irregular pattern on soft materials by photon induced buckling or wrinkling [10] or by the relaxation of the stress in the polymer surface [11]. The pattern size is, however, with 10 to $100 \mu \mathrm{m}$ much larger compared to typical LIPSS.

Some changes of the chemical structure of the irradiated polymer with LIPSS have been observed by Raman spectroscopy [12]. Further the change of the conductivity in the 
LIPSS of P3HT (Poly(3-hexylthiophene-2,5-diyl)) was measured and confirmed that some structural changes happens simultaneously to LIPSS formation below ablation.

The current question is related to the ripples formation in thin polymer films by ns UV laser irradiation using different confinement scenarios. The basic goal is to achieve hierarchical organized pattern by combining bottom-up and topdown patterning approaches only with lasers. Therefore, several experiments were performed to study the formation of sub-micrometer LIPSS by mask projection with excimer laser pulses.

\section{Experimental}

For the experiments silicon wafer $(<100>$ silicon wafer with native oxide) were cleaned with isopropanol. On top of the wafer $\mathrm{a} \sim 710 \mathrm{~nm}$ thick photoresist film (MA P1205; micro resist technology, novolac based photo resist (NPR)) was spun-on at $1500 \mathrm{rpm}$ and tempered subsequently for $2 \mathrm{~min}$ on a hot plate at a temperature of $115^{\circ} \mathrm{C}$. The film features a low defect density due to the high quality filtering of the resist. The film thickness was measured optically by spectroscopic reflectometry applying the optical parameter of the resist (provided by the provider) for fitting the model to the measurements.

These NPR samples were irradiated with excimer laser (LPX 220i from Lambda Physik) beam with a wavelength of $248 \mathrm{~nm}$ and a pulse length of approx. $25 \mathrm{~ns}$. After shaping and homogenizing, the beam is partial polarized $(>75 \%)$ and illuminates a mask that was projected by an $\mathrm{x} 15$ Schwarzschild objective to the sample surface. Different masks were applied for laser irradiation. A variable aperture enabling a homogeneous illuminated spot with sizes between 2 and $100 \mu \mathrm{m}$ and phase masks [13] for smaller features were used. Here phase masks was used as beam splitter producing first order diffracted beams only which are recombined by the objective resulting a sinusoidal energy density pattern [14] with a period of $\sim 1.25 \mu \mathrm{m}$ in the image plane.

After irradiation the surface structures were imaged by scanning electron microscopy (SEM; Ultra 55 from Carl Zeiss). The topography across the spot was determined by white light interference microscopy (WLIM; Micromap 512 from ATOS); only selected spots were measured by atomic force microscopy (AFM; Dimension Icon from Bruker).

\section{Results}

The formation of laser-induced periodic structures in thin polymer films was achieved at different spot geometries and film thicknesses in a specific fluence $(F)$ and pulse number $(N)$ range. A typical appearance of a laser-irradiated surface with LIPSS is shown in Fig. 1a where periodically ordered line structures can be seen.

The linear ripples ordered in domains with a size of several micrometers. The ripples show some irregularities like alteration of the period, bifurcation and local defect in particular at the edges of the domains. The period of the pattern was determined from the SEM image by evaluation of the Fourier spectrum. Spatial periods between 205 and $225 \mathrm{~nm}$ were found for irradiation areas of $100 \times 100 \mu \mathrm{m}^{2}$. Further, the orientation of the ripples was in relation to the irradiation geometry (orientation of the whole array of irradiated spots

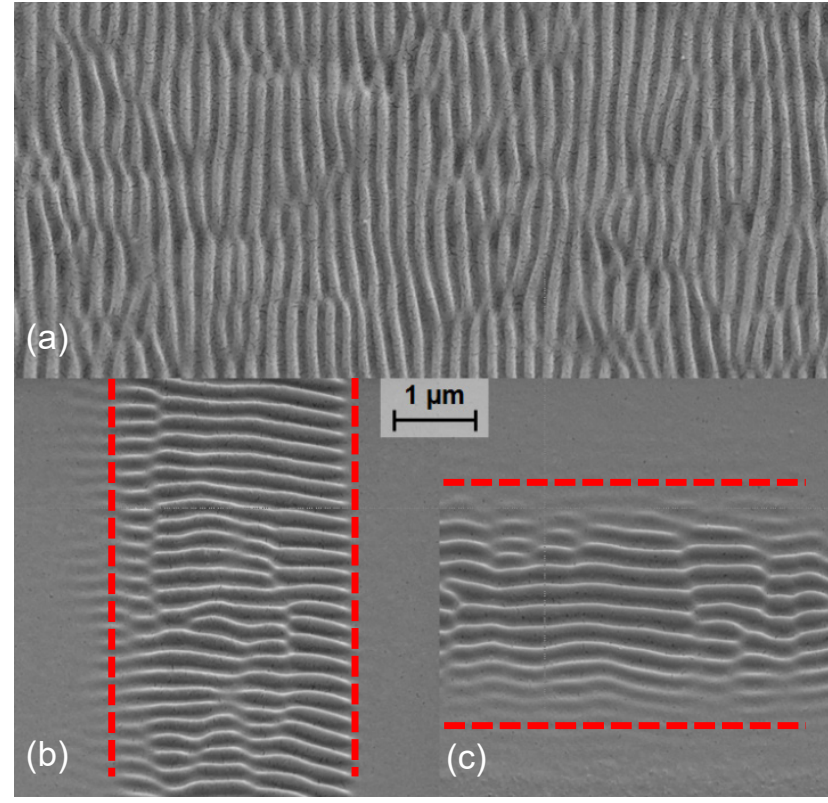

Fig. 1 SEM images of characteristic surface patterns in the photoresist film after Excimer laser irradiation with different masks. a) large area $(100 \times 100 \mu \mathrm{m}$; $\left.F=21.5 \pm 2 \mathrm{~mJ} / \mathrm{cm}^{2}, N=1000\right) ; \mathrm{b}$ ) vertical and c) horizontal slit mask $(100 \mu \mathrm{m} \times 3 \mu \mathrm{m}$; $\left.F=26.5 \pm 2 \mathrm{~mJ} / \mathrm{cm}^{2}, N=1500\right)$. The laser irradiated areas (edges) are shown for small spots in $\mathrm{b}$ and $\mathrm{c}$ too.

as well as the mask edges) almost the same for all different experiments and laser irradiation conditions. The maximal height of the LIPSS determined by AFM was found to be approximately 50 to $70 \mathrm{~nm}$. Except for the very beginning of the ripples growth (depending on fluence and pulse number) almost the same height was determined.

\subsection{Ripples in small laser exposure areas}

The formed LIPSS patterns at small areas defined by $3 \times 100 \mu \mathrm{m}^{2}$ mask are shown in Fig. $1 \mathrm{~b}$ and c. The orientation of the ripples is maintained despite the fact that the imaged mask is changed in size and shape. In particular a rectangular slit mask with a main extension in $\mathrm{x}$ or $\mathrm{y}$ direction was used, which can provide some energy density modulations at the edges of the irradiated field too.

The homogeneity of the LIPSS seems to be better in the case that the mask edges orthogonal to the ripples. This might, as mentioned before, be the result of the diffraction pattern from the edges of the mask. The periods of the LIPSS perpendicular to the mask edges seem to be slightly shorter compared to the parallel orientation; however, both values are in the typical range for the periods.

Further, phase mask irradiation of the photoresist films were performed for further increase the confinement of the LIPPS forming area. The phase mask irradiation causes the formation of interference pattern with a sinusoidal modulation of the energy density with a period of $\sim 1.25 \mu \mathrm{m}$ (see Fig. 2). An overview of the observed features achieved with the phase mask is given in Table 1. With increasing laser fluence up to the ablation threshold different morphologies were found which are listed in table 1. Images of the characteristic surface topographies are depicted in Fig. 2. 


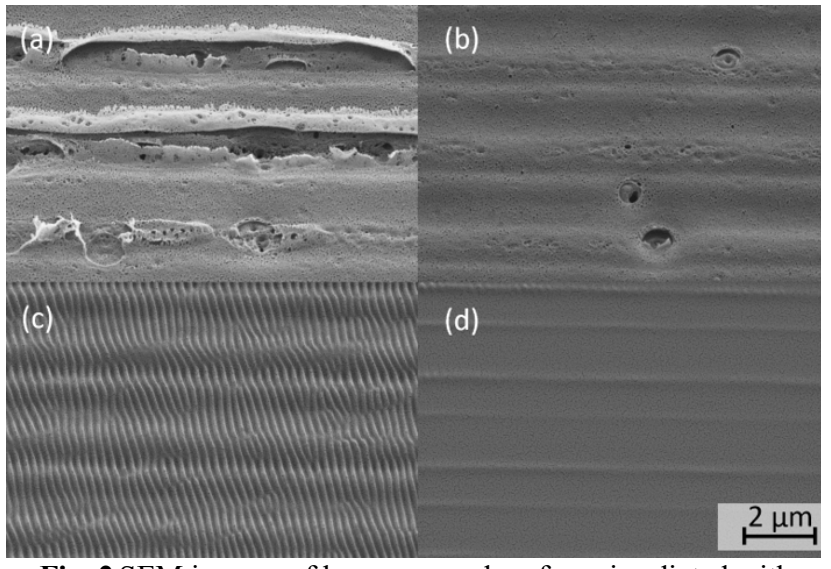

Fig. 2 SEM images of laser exposed surfaces irradiated with $N=1500$ pulses at different laser fluencies $F$ of a) $32.5 \pm 2.5$, b) $28.5 \pm 2$, c) $18 \pm 1.5$ and d) $9.9 \pm 1$ $\mathrm{mJ} / \mathrm{cm}^{2}$. The fluencies used here are the maximum fluence of the sinusoidal energy density distribution.

Table 1 Overview on the observed surface features after excimer laser irradiation at different laser fluences and laser pulse numbers. NPR-thickness: $710 \mathrm{~nm}$.

\begin{tabular}{|c|c|c|c|c|c|c|c|c|c|}
\hline Fluence & \multicolumn{7}{|c|}{ Pulse number $N$} \\
\hline & 1 & 10 & 100 & 300 & 500 & 800 & 1000 & 1200 & 1500 \\
\hline $32.5 \pm 2.5$ & & & & & & & & & \\
\hline $28.5 \pm 2$ & & & & & & & & & \\
\hline $24.5 \pm 2$ & & & & & & & & & \\
\hline $21 \pm 2$ & & & & & & & & & \\
\hline $18 \pm 1.5$ & & & & & & & & & \\
\hline $13 \pm 1$ & & & & & & & & & \\
\hline $12 \pm 1$ & & & & & & & & & \\
\hline $9.9 \pm 1$ & & & & & & & & & \\
\hline $8 \pm 0.5$ & & & & & & & & & \\
\hline
\end{tabular}

Red-NPR ablation; Orange-NPR melting with porous, holes and bubbles on top; Green-LIPSS formation; Light green-NPR modification

Firstly near $8 \mathrm{~mJ} / \mathrm{cm}^{2}$ the swelling of the NPR surface was observed in the areas with the highest laser exposure of sinusoidal energy density distribution. Increasing the fluence to $21 \mathrm{~mJ} / \mathrm{cm}^{2}$ ripples are observed without almost any appearance of ablation. The average height of the ripples area in relation to the not irradiated area was determined to be $50-70 \mathrm{~nm}$ at the edges of the irradiated area by AFM measurement. A further increase of the laser power first sings of resist decomposition/degradation was observed which enhance with further increase of the fluence. The delamination of a very thin film from the surface is observed at fluence of $32.5 \mathrm{~mJ} / \mathrm{cm}^{2}$ that features also small holes as seen in Fig. 2(a).

Further results show that the ripples appear at the same conditions (fluence range of $12-21 \mathrm{~mJ} / \mathrm{cm}^{2}$ and pulse number range of $100-1500$ ) at very different shapes and sizes of the irradiated area.

\subsection{LIPSS at optimized confinement and laser expo- sure conditions}

To further boost the confinement of the interaction, phase mask irradiation was studied in more detail by changing the laser irradiation. In particular, the pulse number and the fluence were adjusted to values that enable the LIPSS formation only in a narrow heated strip.

By tuning the laser irradiation parameters to a particular point using the phase mask pattern consisting of only single dots were achieved. This means, that lateral ordered dot pattern with a size of approx. $240 \mathrm{~nm}$ can be fabricated at particular predefined surface areas.

Due to the narrow laser-heated strip, the LIPSS can be formed only in this volume due to the laser heating related viscosity reduction of the NPR. In the SEM image some material loss next to the formed dot line seems to happen. As seen in the inset, the dots are arranged also in domains; this was concluded also from the FFT (Fast Fourier Transformation) transformation results where the period of the LIPSS are locally changed.

With increasing the fluence the dot chain are widened and change to ripples like pattern. Interestingly the ripples growing in width with increasing laser fluence and forming complete patterned area.

\section{Discussion}

Across all experiments the observed periodic pattern have an experimentally determined period in the range of 200 to $250 \mathrm{~nm}$ and corresponds therefore to the theoretical conditions for LIPSS formation by interference of an incidence with a scattered beam [6]. In contrast to former results here the ripples were observed with a multimode, partially polarized excimer laser beam. As the scattering depends on the polarization, only parts of the beam can contribute to the feedback mechanism for LIPSS formation.

However, several basic questions appear that has to be discussed which are (i) the appearance of LIPSS at very small beam size, (ii) the homogeneity of the photoresist film, (iii) the independence of the pulse number for LIPSS appearance from the spot size.

Interestingly ripples were found only at laser fluencies below laser ablation threshold. The reason of disappearance of the ripples as slightly higher fluencies can be related to thermal processes or near-surface modifications due to the UV light-induced NPR modification.

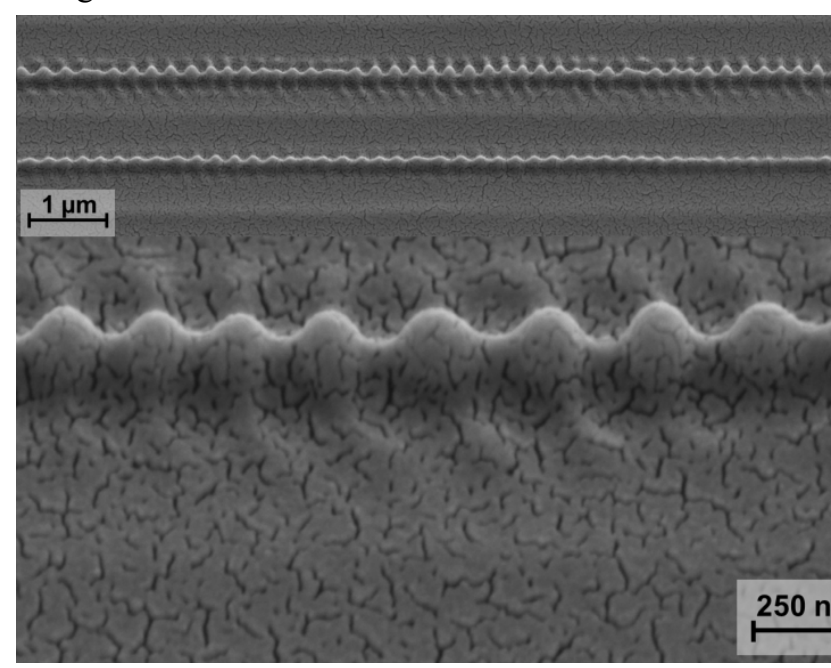

Fig. 3 SEM images of dot chain pattern fabricated by confined generation of LIPSS at $248 \mathrm{~nm}$ within one energy density peak of the phase mask line pattern (similar to one line in Fig. 2) at $F=12.5 \pm 1 \mathrm{~mJ} / \mathrm{cm}^{2}$, $N=1500$. 
The temperature rise within one laser pulse can be estimated from the laser irradiation conditions, the film thickness and the properties of the film material. For phenolic resin (novolac) a thermal diffusivity of $1.3 \times 10^{-7} \mathrm{~m}^{2} / \mathrm{s}$ results in a thermal diffusion length of some $100 \mathrm{~nm}$ during the laser pulse. The optical penetration depth of about $280 \mathrm{~nm}$ has been determined from UV-vis transmission measurements. Due to the low thermal diffusivity the temperature of the photoresist can be estimated from caloric considerations at the end of the pulse.

With that an increase of the resist temperature after the laser pulse by approx. $180 \mathrm{~K}$ are estimated at an energy density of $5 \mathrm{~mJ} / \mathrm{cm}^{2}$. In consequence, the laser-induced temperature exceeds the limit for resist baking of approx. $120^{\circ} \mathrm{C}$ that is given by the provider. Therefore, it can be assumed that the resist has a reduced viscosity due to heating above the glass transition temperature $(T g)$. The viscosity reduction due to laser heating is the main reason allowing mass transport at these low laser fluences below the ablation limit.

The cooling time below $T g$ after the pulse determines the probability that formed LIPSS or roughness can survive as for too long liquid times the surface tension can equalize any surface topography. Therefore, higher fluences probably also allow the formation of some scatter related energy density fluctuations but the surface tension equalizes all roughness due to the longer liquid times. On the other hand long liquid times favor dewetting processes as some time for liquid transport is required. Further, commercial resists can suffer from cross linking due to heat, UV or high energy particle irradiation. Such cross linking can be expected for novolac tempered at temperatures exceeding $160^{\circ} \mathrm{C}$. The modification of polymers due to high energy photon, electron or ion irradiation is well known and can result in chemical and structural changes of the polymer. In particular, the cross linking of the polymer due to the irradiation is a usually observed effect that can be used in the case of novolac photoresist in thermal stabilization of the photoresist. Therefore, high temperatures at laser ablation can change the thermal and viscos properties of the resist film so that LIPSS cannot be achieved anymore.

The small holes observed in the film near the ablation threshold at high pulse number and the thin delaminated film from the surface of the irradiated zones (see Fig. 2 (a) and (b)) are related to laser-induced material modifications. The chemical composition of the NPR includes DNQ (Diazonaphthoquinone) as a photosensitizer that decomposes during UV exposure or heating and forms nitrogen. The small observed holes can result from nitrogen bubbles that were formed by agglomeration of the solved nitrogen. The bubble features were also observed near the ablation threshold but with lower pulse numbers. Such nitrogen formation can be also initiated during the lithographic process by UV photons or thermal treatment [15]. The thin delaminated surface layer seen in Fig. 2 a features also holes and is bended probably due to internal stress. Both the formation of a surface film from a homogeneous spun on polymer as well as the stress related bending can be discussed in the formation of a thin surface modified film due to laser irradiation.

The appearance of the ripples at similar irradiation conditions for different spot sizes is somehow surprising as the probability of scattering at inherent inhomogeneities in the
NPR should change with the spot size. Therefore, with smaller spot size and in consequence smaller laser irradiated area a higher laser pulse number is expected to generate a feedback mechanism for the LIPSS formation. However, if assumed that the dominant scattering centers (stress, holes, bubbles) are generated in the NPR due to the laser irradiation the scattering probability should be independent from the laser irradiated area. The scattering centers which are formed inside the NPR during the laser pulse irradiation can be small nitrogen bubbles which agglomeration later to holes seen in Fig. 2.

The appearance of dot- line pattern LIPSS (see Fig. 3) are somehow unique and be related to the confined laser heating. In relation to the standard theory of LIPSS formation by scattering at a rough surface and superposition of the incidence and a scattered wave the size of the scattering center are rather small and may not induce significant energy density modulations. However, the material transport process for dot formation can be supported by additional hydrodynamic processes that involved in dewetting, Marangoni effects or Rayleigh instabilities. In both cases equilibrium pattern of the respective process may not be achieved due to the time limitation of the melting dynamics.

\section{Conclusion}

The formation of LIPSS due to surface scattering at near surface developed roughness, here probably small bubbles due to the decomposition of the DNQ of the novolac photoresist, has been shown. The LIPSS has been identified as regular ripples due to their period that fits to the period of the surface scattering at roughened surfaces. The confinement of the laser irradiation by using phase mask projection results in additional spatial limitation of the conditions for the LIPSS formation process. At well justified conditions the grating-like ripples reduced to dot pattern with a size of approx. $250 \mathrm{~nm}$ that are aligned along the energy density maxima generated by the phase mask. The dot pattern formation is accomplished by hydrodynamic processes causing mass transport and surface instabilities. This result shows the laser-induced guiding of self-organized pattern in a thin film and in general the benefits of combining top-down and bottom-up processes for high resolution patterning by lasers.

\section{Acknowledgments}

The authors like to thank Mr. Liebeskind for his help in the preparation of the photoresist films and Mrs. Schönherr for the UV measurements. The extensive SEM characterization by Ms. Haferkorn is highly acknowledged. This work was financially supported in parts by the "National Natural Science Foundation of China for International (Regional) Cooperation Research Project (No.11761131015)" and the China Scholarship Council financial support.

\section{References}

[1] T. Lippert, Adv Polym Sci 168 (2004) 51.

[2] P. E. Dyer, S. D. Jenkins, J. Sidhu, Appl. Phys. Lett. 49 (1986) 453.

[3] M. Bolle, S. Lazare, Appl. Surf. Sci. 65-6 (1993) 349 . 
[4] P. E. Dyer, R. J. Farley, R. Giedl et al., Appl. Surf. Sci. 96-8 (1996) 537.

[5] M. Csete, Z. Bor, Appl. Surf. Sci. 133 (1998) 5.

[6] J. E. Sipe, J. F. Young, J. S. Preston et al., Phys. Rev. B 27 (1983) 1141.

[7] H. Niino, M. Nakano, S. Nagano et al., Appl. Phys. Lett. 55 (1989) 510.

[8] T. Bahners, E. Schollmeyer, Angew. Makromol. Chem. 151 (1987) 39.

[9] N. Bityurin, E. Arenholz, N. Arnold et al., Phys. Rev. E 75 (2007) 8.

[10] J. W. Cong, J. J. Wang, J. X. Xie et al., Langmuir 34 (2018) 4793.

[11] F. Bauer, U. Decker, K. Czihal et al., Progress in Organic Coatings 64 (2009) 474.

[12] E. Rebollar, M. Castillejo, T. A. Ezquerra, Eur Polym J 73 (2015) 162.

[13] K. Zimmer, R. Böhme, A. Braun et al., Appl. Phys. A 74 (2002) 453.

[14] P. E. Dyer, R. J. Farley, R. Giedl, Opt. Commun. 115 (1995) 327.

[15] J. Dunkel, F. Wippermann, A. Bruckner et al., Opt. Express 20 (2012) 4763.

(Received: December 17, 2018, Accepted: April 21, 2019) 